\title{
Cylindrical, periodic surface lattice-Theory, dispersion analysis, and experiment
}

\author{
I. V. Konoplev, ${ }^{1, \text { a) }}$ A. J. MacLachlan, ${ }^{2}$ C. W. Robertson, ${ }^{2}$ A. W. Cross, ${ }^{2}$ and A. D. R. Phelps ${ }^{2}$ \\ ${ }_{1}^{1}$ JAI, Department of Physics, University of Oxford, Keble Road, Oxford OXI 3RH, United Kingdom \\ ${ }^{2}$ Department of Physics, SUPA, University of Strathclyde, Glasgow G4 ONG, United Kingdom
}

(Received 6 August 2012; accepted 10 September 2012; published online 20 September 2012)

\begin{abstract}
A two-dimensional surface lattice of cylindrical topology obtained via perturbing the inner surface of a cylinder is considered. Periodic perturbations of the surface lead to observation of high-impedance, dielectric-like media and resonant coupling of surface and non-propagating volume fields. This allows synthesis of tailored-for-purpose "coating" material with dispersion suitable, for instance, to mediate a Cherenkov type interaction. An analytical model of the lattice is discussed and coupled-wave equations are derived. Variations of the lattice dispersive properties with variation of parameters are shown, illustrating the tailoring of the structure's electromagnetic properties. Experimental results are presented showing agreement with the theoretical model. (C) 2012 American Institute of Physics. [http://dx.doi.org/10.1063/1.4754572]
\end{abstract}

One of the important and challenging problems is to understand the phenomena associated with excitation and evolution of electromagnetic (EM) fields on the surface of artificial periodic structures. ${ }^{1-4}$ The studies of surface lattices, which mediate the interaction between active media and EM fields, are an active and exciting research field, recently boosted by development of nanotechnology and understanding of utilizing surface fields (SFs) for compact sources of coherent radiation (CSCR) such as the SPASAR. ${ }^{4}$ Here, we consider the hollow cylindrical structure made from a perfect conductor with the inner surface having two-dimensional (2D) chessboard patterns (periodic perturbations). ${ }^{2,5-7}$ The periodic perturbations manufactured via machining of the smooth surface of the conductor form the square lattice, which geometrically results in localization of the free electrons inside areas which were not machined (scatterers). Thus, electrons move freely inside the scatterers but electron transmission through the whole lattice is terminated, with no electron transport across the lattice in the azimuthal and longitudinal directions, for some range of frequencies. The structure at these frequencies can be considered as a cylindrical waveguide lined with a thin (2D) dielectric ${ }^{8}$ or a highimpedance surface, ${ }^{9,10}$ inside which the excitation of SFs and resonance coupling between surface and near cut-off volume fields (VFs) take place. Also, the topology of the structure is perfect for a number of applications including CSCR and wake-field acceleration. The lattice has a large surface area (in comparison with $\lambda^{2}$, where $\lambda$ is the operating wavelength) and formed by the ensemble of individual scatters which has to be synchronized to observe a coherent state. To resolve the problem, feedback (realized in the setup (Fig. 1) similar to a Fabry-Perot (FP) cavity with a periodic surface lattice (PSL) playing the role of one of the mirrors) can be used. ${ }^{11}$ In Figure 1, conformal mapping is required to observe a cylindrical system from a planar system as shown. The cut-off wave of the cylindrical waveguide (standing wave in a planar system) ensures the feedback and

${ }^{a)}$ Email: ivan.konoplev@physics.ox.ac.uk. Tel.: +44(0)-1865-273456. synchronization of the individual scatterers while the surface fields can be used to interact with active media to observe a CSCR. ${ }^{2,4-8,10-14}$ In this letter, we present an analytical model of PSL eigenmodes defined via coupling of the partial localized SFs with the azimuthally symmetric, near cut-off VFs and comparing the experimental data observed with our understanding of the phenomena. We show that dispersive properties of the structures can be tailored to mediate different phenomena including a Cherenkov instability. ${ }^{8} 10$ The model is defined using a modal approach and a set of coupled wave equations describing the eigenfields' distribution inside the lattice. The eigenfield is considered as a superposition of partial localized SFs and VFs of the unperturbed, partially loaded with a thin dielectric, cylindrical waveguide. Using coupled wave equations derived from the Maxwell equations, the eigenfields' dispersion relations are derived. We note that without the 2D patterns the SF will not be formed; a change of the perfect conductor surface into a high-impedance (dielectric-like) surface can only be observed under specific conditions (see Refs. 1, 8-10 and 12), which is outside the scope of this paper.

Let us consider a $2 \mathrm{D}$ cylindrical structure formed by a chessboard, ${ }^{6,8}$ surface lattice on the inner wall of a hollow, cylindrical, copper waveguide. The pattern's depth, i.e., the individual element amplitude is smaller than the operating wavelength. The resonant coupling (scattering) and cavity eigenmode formation take place if the resonance conditions are satisfied. ${ }^{2,6,8,12-14}$ The discussion and experiments were carried out in the $\mathrm{GHz}$ frequency range ${ }^{6}$ but as will be clear, the equations observed are applicable to $\mathrm{THz}$ and infrared frequencies.

Starting with $\operatorname{curl} \vec{E}=i k \vec{H}-\left(4 \pi \vec{J}_{m} / c\right) ; \operatorname{curl} \vec{H}=-i k \vec{E}$, where $c$ is the speed of light, $\vec{k}=\omega / c$ is the wave vector, $\omega$ is the angular frequency, $\vec{J}_{m}$ is the surface magnetic current, ${ }^{12-14}$ we present the fields as a superposition of partial modes whose transverse structure coincides with eigenmodes of a cylindrical waveguide having a high surface impedance (partially loaded with dielectric). The field's components are $\vec{H}=\sum_{q} C_{q}(z) \vec{H}_{q} ; \vec{E}=\sum_{q} C_{q}(z) \vec{E}_{q}$ (see Refs. 12-14), the 


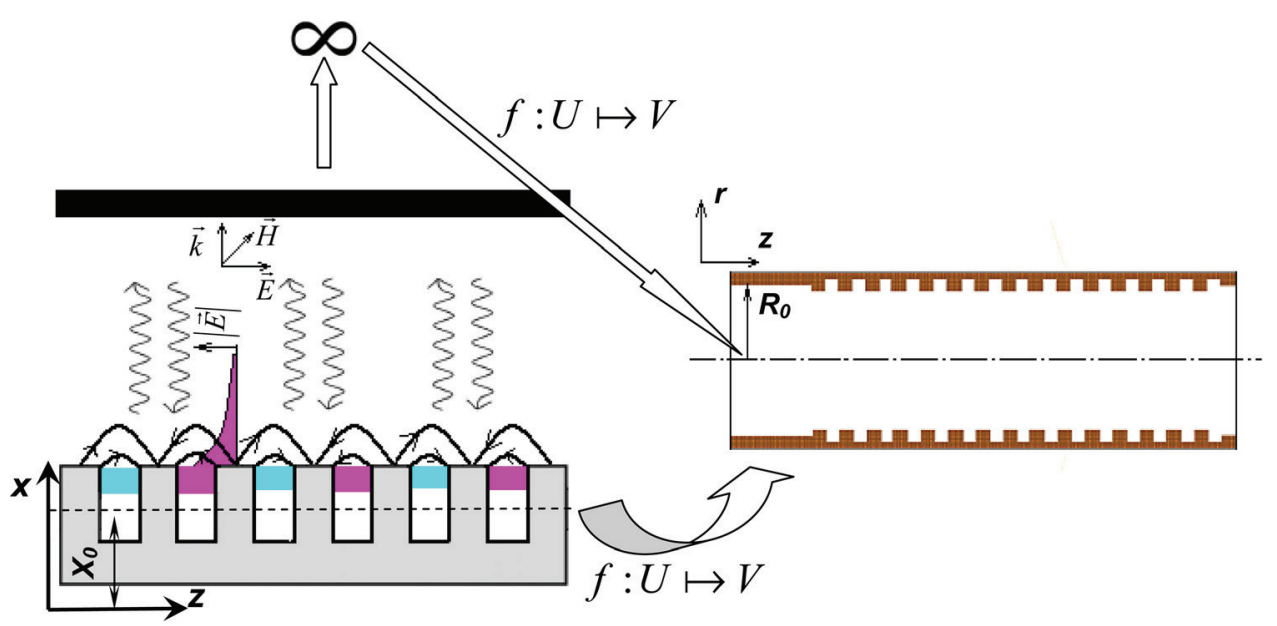

FIG. 1. Schematic showing a planar Fabry-Perot like cavity with one of the mirrors having a high-impedance surface which allows coupling of surface fields and volume fields. The arrows indicate the mathematical steps required to move from planar geometry to a cylindrical cavity with a high-impedance surface similar to a dielectric layer.

$C_{q}(\mathrm{z})$ are the slowly varying amplitudes along the $\mathrm{z}$ coordinate and the wave equations are

$$
\sum_{q} \Delta_{z} C_{q}(z)+\sum_{q}\left(\frac{\omega^{2} \mp\left(\omega_{0}^{q}\right)^{2}}{c^{2}}\right) C_{q}(z)=\left.i \frac{1}{N_{q}} \int_{\sigma} \vec{J}_{m} \vec{H}_{-q}\right|_{r_{0}} d \sigma .
$$

The contour integral taken along the unperturbed cylinder cross-section, $N_{q}^{-1}=4 \pi k /\left(c \int_{\sigma} \vec{H}_{q} \vec{H}_{-q} d \sigma\right)$ is the wave norm $^{12-14}$ and $d \sigma$ defines the integration surface while the lattice is substituted with the set of surface currents, which exist on the waveguide wall $\left(r=r_{0}\right)$. Equation (1) was obtained taking into account: $k^{2}=\omega^{2} / c^{2} ; \Delta=\Delta_{z}+\Delta_{\perp}$ and $\left(\Delta_{\perp} \pm \frac{\left(\omega_{0}^{q}\right)^{2}}{c^{2}}\right) H_{q}=0$, where " + " corresponds to the volume $\left(\omega_{0}^{q}=\omega_{0}^{v}=k_{\perp}^{v} c, k^{2}=k_{z}^{2}+k_{\perp}^{2}\right)$ and “-” to the surface $\left(k^{2}\right.$ $\left.=k_{z}^{2}+(i p)^{2}, \omega_{0}^{q}=\omega_{0}^{s}=p c\right)$ localized modes. Introducing $\Omega^{2}=\left(\left|\omega_{0}^{v}\right|^{2}+\left|\omega_{0}^{s}\right|^{2}\right) / 2, \quad \Phi^{2}=\left(\left|\omega_{0}^{s}\right|^{2}-\left|\omega_{0}^{v}\right|^{2}\right) / 2, \quad \delta=\omega-\Omega$ and considering $\Phi^{2} / \Omega^{2} \ll 1$ (partial fields coupling with $k_{z} \rightarrow 0$ inside a long lattice), using Fourier decomposition (structure is periodic along $\mathrm{z}$ ), the set of equations defining the coupling of the surface field's spatial harmonic with an index $n$ and the fundamental harmonic of the VF can be obtained

$$
\begin{aligned}
& \left(\frac{\partial^{2}}{\partial z^{2}}+2 \bar{K}^{2}\left(\frac{\bar{\delta}}{\bar{K}}+\frac{\bar{\delta}^{2}}{2 \bar{K}^{2}}+\frac{\Delta^{2}}{2 \bar{K}^{2}}\right)\right) A(z)=\left.i \frac{1}{N_{v}} \int_{\sigma} \vec{J}_{m} \vec{H}_{-q}^{v}\right|_{r_{0}} d \sigma \\
& \left(\frac{\partial^{2}}{\partial z^{2}}+2 i n \bar{k}_{z} \frac{\partial}{\partial z}+2 \bar{K}^{2}\left(1-\frac{n^{2} \bar{k}_{z}^{2}}{2 \bar{K}^{2}}+\frac{\bar{\delta}}{\bar{K}}+\frac{\Delta^{2}}{2 \bar{K}^{2}}+\frac{\bar{\delta}^{2}}{2 \bar{K}^{2}}\right)\right) \\
& \times B_{n}(z)=\left.\frac{i}{N_{s}} \int \vec{J}_{m} \vec{H}_{-q}^{s}\right|_{r_{0}} e^{-i n \bar{k}_{z} z} d \sigma
\end{aligned}
$$

where " $s$ " and " $v$ " indicate the norms of the surface and volume fields, $A(\mathrm{z})$ and $B_{n}(\mathrm{z})$ are the slowly varying amplitudes, $\bar{K}=\Omega / c, \Delta=\Phi / c$, and $\bar{\delta}=\delta / c$. The right-hand sides of Eqs. (2a) and (2b) can be rewritten as $\alpha_{v, s}^{2} F_{s, v},{ }^{12-14}$ where $\alpha_{v, s}$ are the coupling coefficients and $F_{s, v}$ are functions of the amplitudes $A(\mathrm{z})$ and $B_{\mathrm{n}}(\mathrm{z})^{12-14}$ (i.e., Eqs. (2a) and (2b) are coupled only if $\alpha_{v, s}^{2} F_{s, v} \neq 0$ ). Here, we will treat $\alpha_{s, v}$ as variable parameters, the strict definition of which is outside the scope of this letter and can be found using the technique discussed in Refs. 12-14. The coupling between the near cutoff, VF fundamental harmonic, and SF takes place if the SF harmonic's number $n$ coincides with the harmonic of the lattice $\bar{n}$. Assuming that the lattice is sinusoidal $(n= \pm 1)$, introducing $Z=\bar{K} z, \quad \hat{A}_{0}(Z)={\underset{\sim}{\delta}}_{0}(Z) / \alpha_{\nu}, \quad \hat{B}_{ \pm}(Z)=B_{ \pm}(Z) / \alpha_{s}$, $\alpha^{2}=\alpha_{v} \alpha_{s} / \bar{K}^{2}, \quad \Gamma_{\tilde{\sigma}}=\bar{k}_{z} / \bar{K}, \quad \tilde{\delta}=\bar{\delta} / \bar{K}=(\omega-\Omega) / \Omega ; \tilde{\tilde{\Delta}}=\Delta / \bar{K}$ $\ll 1, \omega_{e}^{2}=2 \tilde{\delta}+\left(\tilde{\delta}^{2}+\tilde{\Delta}^{2}\right)$ and assuming that $\hat{A}_{0}, \hat{B}_{ \pm}$are proportional to $e^{i \Lambda Z}$, the dispersion relation can be found

$$
\begin{aligned}
& \left(\omega_{e}^{2}-\Lambda^{2}\right)\left[\Lambda^{4}-2 \Lambda^{2}\left(2+\Gamma^{2}+\omega_{e}^{2}\right)+\left(2-\Gamma^{2}+\omega_{e}^{2}\right)^{2}\right] \\
& \quad=2 \alpha^{4}\left(2-\Gamma^{2}+\omega_{e}^{2}-\Lambda^{2}\right) .
\end{aligned}
$$

The detuning parameter $\Gamma$ is a function of the structure's geometry (for a specific mode) as it links the periodicity of the lattice and $\omega_{0}^{s}=p c$ and $\omega_{0}^{v}=k_{\perp}^{v} c$. One notes that if $\alpha \rightarrow 0$, no coupling is observed (Fig. 2, broken lines) while large $\alpha$ such as $\alpha=1$ is associated with strong coupling and the whole dispersion is affected (right insets of Fig. 2). For clarity reasons only, the dispersions (Fig. 2) were observed for the structure having the same mean diameter as used in experiments reported ${ }^{6}$ under the assumption that $\Delta=0$ and $\alpha$ $\ll 1$ (weak coupling, solid lines). If $\alpha \ll 1$, then the eigenmode dispersion deviates from the unperturbed values in the vicinity of the crossing points where the split between branches takes place (main case). In Figure 2, eigenmodes' dispersions are presented for the set of structure detuning parameter $\Gamma=1, \sqrt{2}$, and 2.3, respectively. With increase of $\Gamma$ from 1 to 2.3 , the crossing points are moving towards the vertex of the parabola ${ }^{8}$ and at $\Gamma=\sqrt{2}$ and $\alpha=0.25$ (Fig. 2(b)), the crossing points merge at the center of the parabola. Also, the gap in this case (as expected) is larger as compared with $\alpha=0.1$. Assuming now a Cherenkov interaction between an electron beam and the eigenfield formed, the synchronism condition required for the interaction to take place is $\omega=2 \pi v_{z} l / d_{z}\left(k_{\mathrm{z}}=0, l=1\right.$ here $l$ is the electron beam harmonic) leading to an electron beam velocity condition $1 / \sqrt{2} \leq v_{z} / c<1$. At $\Gamma=2.3$ and $\alpha=0.1$, the eigenmodes' dispersions (Fig. 2(c)) change further and now the interaction between the electron beam and the EM fields can be observed even at lower beam velocities $1 / \Gamma \leq v_{z} / c,{ }^{8}$ thus making an increase of $\Gamma$ synonymous with increasing effective dielectric refractive index. On the right-side insets 

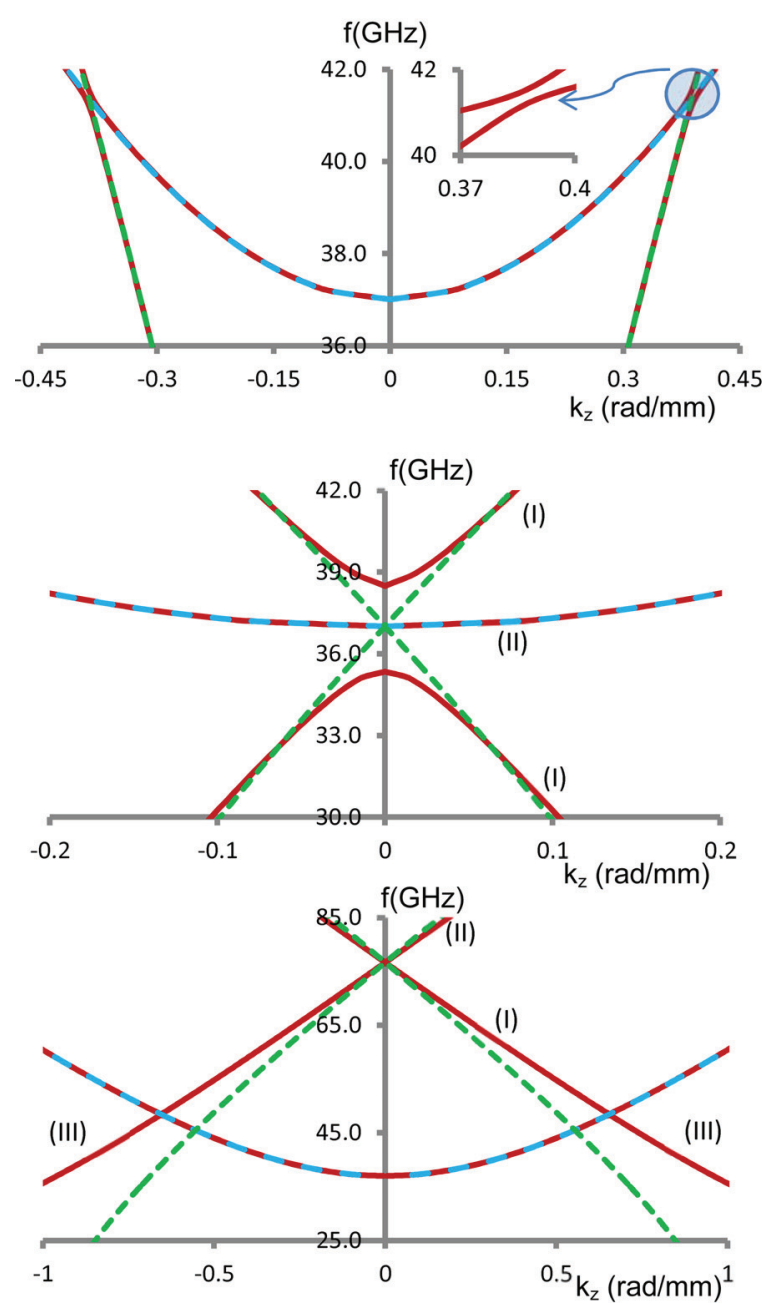

(a)

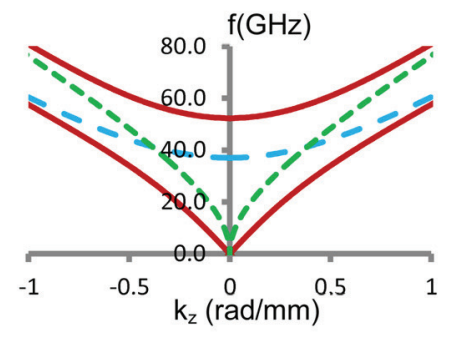

(b)

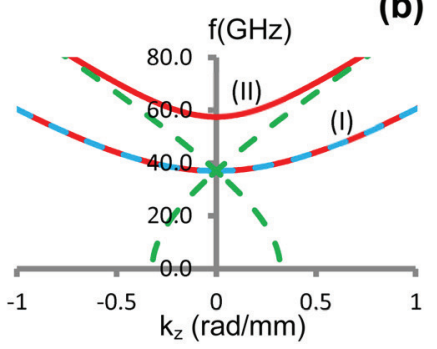

(c)

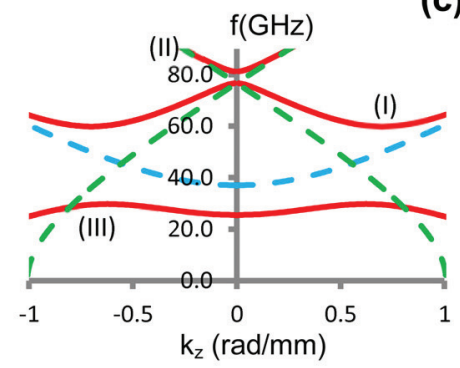

FIG. 2. Dispersion diagrams observed from Eq. (3) for a structure having: mean diameter $7.9 \mathrm{~cm}$, number of azimuthal variation 28 illustrating the coupling between partial volume $\mathrm{TM}_{0,10}$ and surface $\mathrm{EH}_{28,1}$ fields when $\lambda_{\mathrm{c}} / d_{\mathrm{z}}$ and $\alpha$ coupling coefficient $(\alpha=1$ for all right hand-side insets) are: (a) 1 and 0.1 ; (b) $\sqrt{2}$ and 0.25 ; (c) 2.3 and 0.1 , respectively. In all figures, the dashed lines indicate the dispersion of the unperturbed partial fields, i.e., when $\alpha=0$.
(Fig. 2), the dispersion diagrams for $\alpha=1$ are shown and it is clear that these are formed via an increasing gap between branches at the crossings. Analyzing the graphs we see that the positions of the extrema points $\left(\partial f / \partial k_{z}=0\right)$, indicating the positions of the cavity eigenmodes, are different from the unperturbed waveguide cut-off frequencies and having $f / k_{z}<c$ and $\partial f / \partial k_{z}$ with sign variation illustrates the possibility for observing slow forward and backward waves, required for a Cherenkov interaction to take place.

The experiments, for which the set-ups were discussed in Refs. 3 and 6, have been conducted to link theoretical understanding with experimental measurements. The first set of experiments is based on excitation of the structure's eigenmode inside the lattice via resonant scattering of the near cut-off VFs to the SFs. To observe the near cut-off VF, a coaxial line was used and it was terminated at the lattice, resulting in excitation of the near cut-off, azimuthally symmetric mode. The resonant coupling between the fields manifests itself as two gaps (Fig. 3(a)) in the signal transmission observed at small observation angles with respect to the structure's axis of symmetry. The gaps are shifting in the frequency domain with variation of the observation angle. Each minima correspond to a point on the dispersion and the frequency shift corresponds to the move of the point along the dispersions' branches. A specific angle corresponds to a longitudinal wavenumber (ratio $k$ and $k_{\perp}, k_{\perp}^{2} / k^{2}=\varepsilon^{2}$ ). Thus, measuring the frequency shift of the minima and knowing the observation angle one can recover the dispersion. The technique works for small observation angles, corresponding to small variations of $k_{\mathrm{z}}$ around 0 , as at larger angles the appearance of other minima can be expected due to noncoherent scattering ${ }^{3}$ of propagating waves. In Figure 3(a) and the inset of this figure, the results of the transmission measurements for the observation angles $\left[0^{\circ} ; 6^{\circ}\right]$ and the shift of the observed minima are shown. There is correlation between the theory and the predictions, while differences come from the fact that the structure studied in the experiment is of finite size $(4.8 \mathrm{~cm}$ long and $7.9 \mathrm{~cm}$ in diameter), while the theory is developed for an infinitely long structure. In the second set of experiments, we illustrate the high-Q mode excitation ${ }^{6,8}$ at a frequency different from the cut-off frequency of the non-perturbed waveguide. For this purpose, the cavity was driven by short $50 \mathrm{~ns}$ pulses having $5 \mathrm{~ns}$ rise and fall times and formed via amplitude modulation of the high-frequency wave. The structure is based on the waveguide with an unperturbed radius of $3.95 \mathrm{~cm}\left(\mathrm{TM}_{0,10}\right.$ mode cut-off frequency, i.e., $\partial f / \partial k_{z}=0$ is $\sim 37 \mathrm{GHz}$ ), while from the previous experiment it was expected that the cavity eigenmode should be observed at $\sim 37.7 \mathrm{GHz}$, i.e., $\partial f / \partial k_{z}=0$ is above the cut off frequency of the conventional waveguide. The results shown in Fig. 3(b) illustrate the pulse propagation through the structure and cavity 

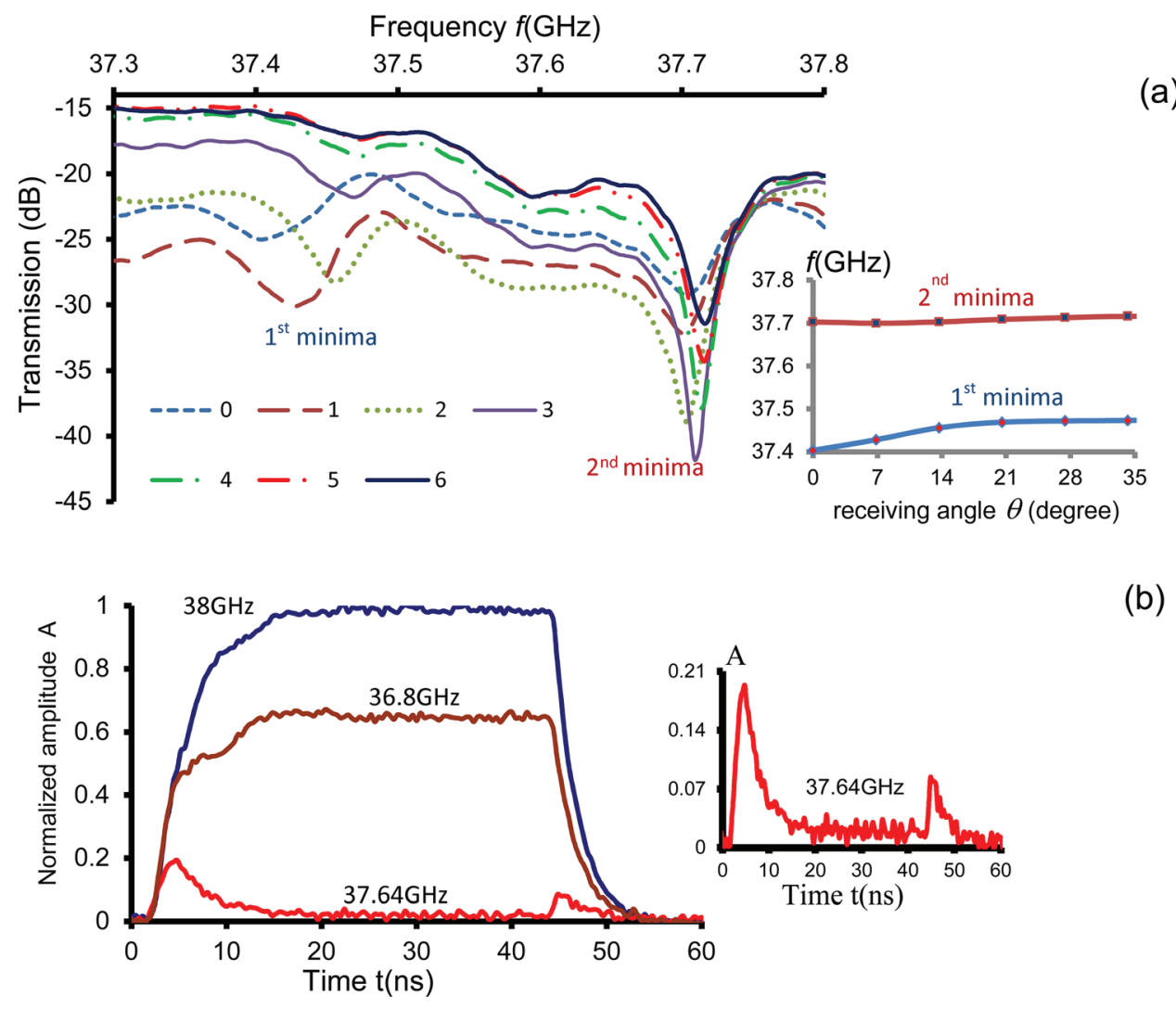

(a)

FIG. 3. The experimental studies of (a) frequency dependence of signal transmission through the cylindrical 2D PSL measured at the set of angles $\theta \in\left[0^{\circ} ; 6^{\circ}\right]$ with right hand side insets illustrating the shifts of the minima measured with variation of observation angle $\theta$, (b) pulse propagation through the 2D PSL measured having carrier frequencies $38.0 \mathrm{GHz}, 37.64 \mathrm{GHz}$, and $36.8 \mathrm{GHz}$, with the right hand-side insets magnifying the results observed for pulses formed with the $37.64 \mathrm{GHz}$ carrier frequency. excitation at $37.64 \mathrm{GHz}$ (inset of Fig. 3(b)). Tuning the signal carrier frequency to $37.64 \mathrm{GHz}$ results in a visible change of the pulse profile, similar to those observed during excitation of critically coupled cavities, confirming our understanding of the structure's dispersion and phenomena observed. The cavity Q-factor can also be estimated from the exponential time-decay observed in Fig. 3(b) and is found to be approximately 600 .

To summarize, in this paper, we have developed an analytical theory of the coupling of partial fields inside a 2D cylindrical periodic structure formed by small periodic perturbations. The surface lattice is described as a highimpedance thin material (dielectric layer) allowing SFs to be taken into consideration as partial fields forming the lattice eigenmode. The dispersion relation describing the EM property of the lattice has been derived and dispersion diagrams were analyzed for different sets of parameters. We have discussed the possibility to excite the structure with a relativistic electron beam to observe Cherenkov interaction. The theory developed was linked to the previous works and analysis was carried out taking into account the parameters of the experimental set-up. We have measured the dependence of the spectra of the transmission coefficient versus angle of observation and discussed the method of deriving the dispersion from the data obtained. The structure has been considered as a cavity and eigenmode excitation using a short pulse has been carried out. We have shown that in spite of the complexity of the measurements a good agreement, between theoretical understanding and experimental measurements, has been observed. We would like to note that the theory developed is applicable for a broad range of frequencies including $\mathrm{THz}$ and infrared frequencies.
The support of the UK EPSRC is gratefully acknowledged.

${ }^{1}$ J. B. Pendry, A. J. Holden, W. J. Stewart, and I. Youngs, Phys. Rev. Lett. 76, 4773 (1996); F. J. Garcia-Vidal and J. B. Pendry, ibid. 77, 1163 (1996); N. Papasimakis, V. A. Fedotov, Y. H. Fu, D. P. Tsai, and N. I. Zheludev, Phys. Rev. B 80, 041102 (2009); D. Yu. Shchegolkov, A. K. Azad, J. F. O'Hara, and E. I. Simakov, ibid. 82, 205117 (2010); V. A. Fedotov, M. Rose, S. L. Prosvirnin, N. Papasimakis, and N. I. Zheludev, Phys. Rev. Lett. 99, 147401 (2007).

${ }^{2}$ I. V. Konoplev, A. D. R. Phelps, A. W. Cross, and K. Ronald, Phys. Rev. E 68, 066613 (2003); I. V. Konoplev, P. McGrane, W. He, A. W. Cross, A. D. R. Phelps, C. G. Whyte, K. Ronald, and C. W. Robertson, Phys. Rev. Lett. 96, 035002 (2006); I. V. Konoplev, L. Fisher, A. W. Cross, A. D. R. Phelps, K. Ronald, and C. W. Robertson, Appl. Phys. Lett. 96, 261101 (2010); I. V. Konoplev, A. W. Cross, and A. D. R. Phelps, IEEE Trans. Plasma Sci. 39, 2610 (2011).

${ }^{3}$ W. L. Barnes, A. Dereux, and T. W. Ebbesen, Nature 424, 824 (2003); N. Papasimakis, V. A. Fedotov, N. I. Zheludev, and S. L. Prosvirnin, Phys. Rev. Lett. 101, 253903 (2008); J. Renger, S. Grafström, and L. M. Eng, Phys. Rev. B 76, 045431 (2007); S. A. Maier, S. R. Andrews, L. MartínMoreno, and F. J. García-Vidal, Phys. Rev Lett. 97, 176805 (2006).

${ }^{4}$ D. J. Bergman and M. I. Stockman, Phys. Rev. Lett. 90, 027402 (2003); M. I. Stockman, Nature Photon. 2, 327 (2008); M. I. Stockman, J. Opt. 12, 024004 (2010).

${ }^{5}$ N. S. Ginzburg, I. V. Konoplev, and A. S. Sergeev, Zh. Tech. Fiziki (J. Tech. Phys. (ZhTP)) 66, 108 (1996); I. V. Konoplev, A. W. Cross, W. He, A. D. R. Phelps, K. Ronald, G. R. M. Robb, C. G. Whyte, N. S. Ginzburg, N. Y. Peskov, and A. S. Sergeev, Nucl. Instrum. Methods Phys. Res. A 445, 236 (2000).

${ }^{6}$ I. V. Konoplev, L. Fisher, K. Ronald, A. W. Cross, A. D. R. Phelps, C. W. Robertson, and M. Thumm, Appl. Phys. Lett. 96, 231111 (2010); I. V. Konoplev, L. Fisher, A. W. Cross, A. D. R. Phelps, K. Ronald, and M. Thumm, ibid. 97, 261102 (2010); A. W. Cross, I. V. Konoplev, K. Ronald, A. D. R. Phelps, W. He, C. G. Whyte, N. S. Ginzburg, N. Yu. Peskov, and A. S. Sergeev, ibid. 80, 1517 (2002).

${ }^{7}$ N. E. Glass, Phys. Rev. A 36, 5235 (1987); P. T. Worthing and W. L. Barnes, Appl. Phys. Lett. 79, 3035 (2001); S. Riechel, C. Kallinger, U. Lemmer, J. Feldmann, A. Gombert, V. Wittwer, and U. Scherf, ibid. 77, 2310 (2000). 
${ }^{8}$ I. V. Konoplev, A. J. MacLachlan, C. W. Robertson, A. W. Cross, and A. D. R. Phelps, Phys. Rev. A 84, 013826 (2011).

${ }^{9}$ D. F. Sievenpiper, E. Yablonovitch, J. N. Winn, S. Fan, P. R. Villeneuve, and J. D. Joannopoulos, Phys. Rev. Lett. 80, 2829 (1998); E. Lier, D. H. Werner, C. P. Scarborough, Q. Wu, and J. A. Bossard, Nature Mater. 10, 252 (2011).

${ }^{10}$ J. T. Shen, P. B. Catrysse, and S. Fan, Phys. Rev. Lett. 94, 197401 (2005); P. B. Catrysse, G. Veronis, H. Shin, J.-T. Shen, and S. Fan, Appl. Phys. Lett. 88, 031101 (2006); M. J. Lockyear, M. J. Lockyear, A. P. Hibbins, and J. R. Sambles, Phys. Rev. Lett. 102, 073901 (2009).

${ }^{11}$ V. L. Bratman, B. S. Dumesh, A. E. Fedotov, P. B. Makhalov, B. Z. Movshevich, and F. S. Rusin, IEEE Trans. Plasma Sci. 38, 1466 (2010); Yu. A. Grishin, M. R. Fuchs, A. Schnegg, A. A. Dubinskii, B. S. Dumesh, F. S. Rusin, V. L. Bratman, and K. Möbius, Rev. Sci. Instrum. 75, 2926 (2004).
${ }^{12}$ A. W. Cross, I. V. Konoplev, A. D. R. Phelps, and K. Ronald, J. Appl. Phys. 93, 2208 (2003).

${ }^{13}$ N. F. Kovalev, I. M. Orlova, and M. I. Petelin, Izv. Vyssh. Uchebn. Zaved., Radiofiz. 11, 783 (1968); I. V. Konoplev, P. McGrane, A. W. Cross, K. Ronald, and A. D. R. Phelps, J. Appl. Phys. 97, 073101 (2005); G. Burt, S. V. Samsonov, A. D. R. Phelps, V. L. Bratman, K. Ronald, G. G. Denisov, W. He, A. R. Young, A. W. Cross, and I. V. Konoplev, IEEE Trans. Plasma Sci. 33, 661 (2005).

${ }^{14}$ G. Burt, S. V. Samsonov, K. Ronald, G. G. Denisov, A. R. Young, V. L. Bratman, A. D. R. Phelps, A. W. Cross, I. V. Konoplev, W. He, J. Thomson, and C. G. Whyte, Phys. Rev. E 70, 046402 (2004); I. V. Konoplev, P. McGrane, A. D. R. Phelps, A. W. Cross, and K. Ronald, Appl. Phys. Lett. 87, 121104 (2005); I. V. Konoplev, G. Doherty, A. W. Cross, A. D. R. Phelps, and K. Ronald, ibid. 88, 111108 (2006). 